\title{
VALIDAÇÄO DA VERSÄO BRASILEIRA DO TESTE DE TRIAGEM DO ENVOLVIMENTO COM ÁLCOOL, CIGARRO E OUTRAS SUBSTÂNCIAS (ASSIST)
}

\author{
Iara Ferraz Silva Henrique, Denise De Micheli, Roseli Boerngen de Lacerda, \\ Luiz Avelino de Lacerda, Maria lucia Oliveira de Souza Formigoni* \\ Trabalho realizado no Departamento de Psicobiologia da Universidade Federal de São Paulo (UNIFESP), \\ São Paulo, SP, e Departamento de Farmacologia da Universidade Federal do Paraná (UFPR), Curitiba, PR.
}

RESUMO - OBJETIVO. Considerando-se os índices cada vez maiores do uso de substâncias psicoativas no Brasil, faz-se necessário um instrumento para a sua detecção precoce que seja válido, confiável e passível de ser utilizado em serviços de atenção primária à saúde. Para tal finalidade, foi desenvolvido um instrumento denominado teste de triagem do envolvimento com álcool, tabaco e outras substâncias (ASSIST), em um projeto multicêntrico coordenado pela Organização Mundial de Saúde (OMS). 0 presente estudo teve como objetivo avaliar as propriedades psicométricas da versão brasileira deste instrumento, sua validade concorrente e confiabilidade na detecção do uso de substâncias psicoativas e problemas associados.

Métodos. 0 ASSIST e três instrumentos diagnósticos validados (MINI-Plus, AUDIT e RTQ) foram aplicados a 99 pacientes que procuraram serviços de assistência primária/secundária à saúde e a
48 pacientes em tratamento especializado para dependência de álcool ou outras substâncias.

Resultados. Os escores do ASSIST para álcool apresentaram boa correlação com os escores do AUDIT. 0 ASSIST apresentou boa sensibilidade e especificidade na detecção de uso abusivo/ dependência de álcool, maconha e cocaína, considerando como padrão-ouro o diagnóstico do MINI-Plus. A confiabilidade do instrumento foi boa (alfa de Cronbach de 0,80 para álcool, 0,79 para maconha e 0,8I para cocaína).

Conclusã̃o. As propriedades psicométricas da versão brasileira do ASSIST se mostraram satisfatórias, o que recomenda a sua aplicação a pacientes de serviços de atenção primária/secundária à saúde.

UnITERMOS. ASSIST-OMS. Validação. Dependência de drogas. Teste de triagem. Álcool.

\section{INTRODUÇÃO}

O consumo de substâncias psicoativas é bastante freqüente em nossa sociedade, variando desde o uso ocasional até a dependência ${ }^{1-4}$. Estudos epidemiológicos têm detectado índices de uso cada vez maiores nos últimos anos, tanto no Brasili,4 como em outras regiões do mundo 5,6 .

O primeiro levantamento domiciliar sobre o uso de drogas no Brasil, realizado pelo CEBRID (Centro Brasileiro de Informações sobre Drogas da UNIFESP) em uma amostra representativa das 107 cidades brasileiras com mais de 200.000 habitantes, detectou índices de | I,5\% para dependência de álcool, 9\% para dependência de tabaco e $1 \%$ para dependência de maconha, sendo que as porcentagens de uso na vida destas substâncias foram de 68,7\%, 41\% e $7 \%$, respectivamente 1,2 . Em geral, os usuários de substâncias psicoativas procuram os serviços

* Correspondência

Rua Botucatu, 862 - Edifício de Ciências Biomédicas $1^{\circ}$ andar - CEP: 04023-062 - São Paulo - SP E-mail:mlformig@psicobio.epm.br Fone: (11) 5539-0155 - Fax: (11) 5572-5092 especializados já em uma fase muito grave do distúrbio ${ }^{7}$. Considerando-se que a intervenção em fases iniciais do problema melhora muito o prognóstico, faz-se necessário o desenvolvimento de estratégias de detecção e intervenção precoces ${ }^{6}$. O primeiro recurso necessário é um instrumento para detecção do uso que seja válido, confíavel e de baixo custo.

Existem alguns instrumentos já validados no Brasil, para triagem da dependência de álcool como o $\mathrm{CAGE}^{8}$ e o AUDIT ${ }^{9,10}$, ou para avaliação da sua gravidade, como o SADD e a ADS'I. Entretanto, não encontramos instrumentos validados para o português falado no Brasil para detecção do uso abusivo ou dependência de outras substâncias.

Sob a coordenação da Organização Mundial de Saúde (OMS), pesquisadores de vários países desenvolveram um instrumento para detecção do uso de álcool, tabaco e outras substâncias psicoativas, denominado ASSIST (Alcohol, Smoking and Substance Involvement Screening Test). Este instrumento foi traduzido para várias línguas, inclusive para o português falado no Brasil, já tendo sido testado quanto à sua confiabilidade e factibilidade, quando aplicado por pesquisadores 6 . $O$ estudo da confiabilidade teste-reteste do ASSIST foi realizado com 236 indivíduos, em diferentes locais do mundo 6 . Naquele estudo foi observada uma boa confiabilidade teste-reteste (coeficientes Kappa entre 0,58 a 0,90 para as principais questões), sendo o seu uso considerado factível em locais de assistência primária à saúde.

Algumas características do ASSIST sugerem que ele seja adequado para uso em serviços de assistência não especializados: sua estrutura padronizada, rapidez de aplicação, abordagem simultânea de várias classes de substâncias, facilidade de interpretação e a possibilidade de ser utilizado por profissionais de saúde de formações diversas. Entretanto, é importante avaliar se suas características psicométricas, calculadas com base em dados coletados em vários países, se mantêm na versão brasileira, adaptada à língua e cultura e também se este instrumento é válido. O objetivo do presente estudo foi avaliar o desempenho da versão brasileira do ASSIST na detecção de uso de álcool e outras drogas em serviços de atenção primária/secundária à saúde, sua consistência interna e validade concorrente. 


\section{Métodos}

Participantes - Este estudo foi parte da fase II do projeto multicêntrico "An International Validity Study of the Alcohol and Substance Involvement Screening Test", organizado pela OMS, que envolveu sete países, com cerca de 150 participantes em cada país. Foi utilizada uma amostra de critério. De acordo com o planejamento inicial, em cada país participante a amostra deveria ser constituída por 100 pacientes oriundos de serviços de assistência primária (SAPS) e 50 de serviços especializados no tratamento de dependências (DEP), seguindo uma estratificação por sexo e idade. Este procedimento de estratificação foi utilizado para assegurar a existência de um número adequado de pessoas de ambos os sexos em cada faixa etária. Foram incluídos pacientes entre 18 e 45 anos, sendo um terço da amostra constituído por mulheres e dois terços por homens. O limite superior de idade (45 anos) foi baseado em estudos anteriores que sugerem ser baixa a prevalência de dependência de drogas acima desta idade. A idade inferior (I 8 anos) foi escolhida para evitar problemas logísticos quanto à obtenção de autorização para participação de pais ou responsáveis. Para possibilitar a comparabilidade de dados entre os diversos países foram consideradas três faixas etárias: 18 a 25, 26 a 35 e 36 a 45 anos. Os participantes dos serviços especializados (DEP) foram selecionados aleatoriamente por sorteio dos registros dos pacientes que se encontravam em tratamento em cada dia, até que fossem atingidas as quotas de cada grupo considerando-se a idade e o sexo.

A amostra brasileira incluiu 147 pessoas, sendo 73 (50\%) em São Paulo e 74 (50\%) em Curitiba. Um grupo de 99 pessoas foi recrutado em serviços de assistência primária/secundária à saúde (SAPS), incluindo unidades básicas de saúde (pertencentes ao Programa de Saúde da Família), ambulatórios e enfermarias não especializados em dependência de substâncias psicoativas (unidades de cardiologia, gastroenterologia, neurologia, pneumologia e ortopedia). Os 48 pacientes que constituíram o grupo de dependentes de álcool e outras drogas (DEP) foram recrutados em serviços ambulatoriais ou de internação especializados no tratamento de drogas, nas cidades de São Paulo, Campinas e Curitiba.
Foi constituída uma amostra estratificada por sexo e idade, sendo que $62 \%$ dos pacientes eram homens e $38 \%$ mulheres. Os pacientes foram também distribuídos em três faixas etárias: 18 a 25 anos (42\% a 48\%)', 26 a 35 anos (30\% a $31 \%)^{\prime}$ e 36 a 45 anos (27\% a 29\%)'.

Foram considerados critérios de inclusão: idade entre 18 e 45 anos; ter proficiência na língua portuguesa, atestada pela capacidade de leitura e compreensão dos questionários e instrumentos de auto-aplicação utilizados; não apresentar retardo mental, sintomas psicóticos ou distúrbios cognitivos ou de comportamento graves; ter disponibilidade para participar de uma sessão de aplicação dos instrumentos com duração média de 90 minutos, não estar intoxicado ou em síndrome de abstinência de álcool ou drogas no momento da entrevista; concordar em participar do estudo e assinar um termo de consentimento informado, aprovado pelos comitês de Ética em Pesquisa da UNIFESP e da UFPR.

Procedimentos - Os participantes foram convidados a participar do estudo através de abordagem direta, pelos pesquisadores, nos locais acima mencionados, tendo sido obtida autorização prévia dos responsáveis por cada serviço. Cada participante recebeu uma ajuda de custo de $R \$ 20,00$, para ressarcimento de despesas de alimentação e transporte. A entrevista, incluindo a aplicaçãa de todos os instrumentos e a explicação sobre os objetivos do projeto, durou em média 90 minutos. Os seguintes instrumentos foram aplicados pelo entrevistador:

Formulário de informações demográficas - questionário no qual foram coletadas variáveis sociodemográficas, incluindo estado civil, religião, raça, situação ocupacional e educacional.

ASSIST (Anexo I) - questionário estruturado contendo oito questões sobre o uso de nove classes de substâncias psicoativas (tabaco, álcool, maconha, cocaína, estimulantes, sedativos, inalantes, alucinógenos, e opiáceos). As questões abordam a freqüência de uso, na vida e nos últimos três meses, problemas relacionados ao uso, preocupação a respeito do uso por parte de pessoas próximas ao usuário, prejuízo na execução de tarefas esperadas, tentativas mal sucedidas de cessar ou reduzir o uso, sentimento de compulsão e uso por via injetável. Cada resposta corresponde a um escore, que varia de 0 a 4 , sendo que a soma total pode variar de 0 a 20. Considera-se a faixa de escore de 0 a 3 como indicativa de uso ocasional, de 4 a 15 como indicativa de abuso e $\geq 16$ como sugestiva de dependência. A versão em português utilizada foi previamente submetida a processo de tradução e retrotradução, tendo por base a versão final do instrumento, em inglês, desenvolvida na fase I do projeto multicêntrico 6 .

MINI-Plus' ${ }^{12}$, versão traduzida para o português ${ }^{3}$ - instrumento para diagnóstico de problemas psiquiátricos. Foram utilizadas apenas as secções relativas ao uso de álcool (K) e drogas $(L)$, déficit de atenção e hiperatividade (Q), distúrbios de conduta e desordens de personalidade anti-social (W)

Após a aplicação destes instrumentos pelo entrevistador, os seguintes questionários foram entregues para serem preenchidos diretamente pelo paciente:

Alcohol Use Disorders Identification Test (AUDIT) - instrumento de triagem do uso de álcool e de problemas associados;

Revised Fargerstron Tolerance Questionnaire (RTQ) questionário de 10 itens desenvolvido para avaliar dependência de nicotina (tradução nossa do instrumento desenvolvido por Tate \& Schmitz, (993)14.

Análise estatística - A comparação dos grupos SAPS e DEP, em relação às variáveis categóricas, foi realizada pelo teste do $X^{2}$ e para comparação das variáveis intervalares foi utilizado o teste "t" de Student. Em todas as análises realizadas foi adotado o nível de significância de $5 \%$.

Para cada droga analisada foram calculadas a distribuição e a soma total dos escores do ASSIST. Foi considerada "a droga principal", aquela que gerou os escores mais altos para cada pessoa. Foram também calculadas as porcentagens de participantes que obtiveram escore positivo (escores maiores que zero) no ASSIST para cada droga. As médias dos escores máximos do ASSIST dos dois grupos foram comparadas através do teste "t" de Student. Estes cálculos foram realizados incluindo e excluindo álcool e tabaco para permitir avaliar a utilidade do ASSIST, tanto na triagem geral do uso de drogas como na triagem de drogas de uso ilícito. Para avaliar a relevância de cada questão foram calculados as porcentagens de pacientes que nela pontuaram e os escores médios atingidos em cada questão. 
Anexo I - ASSIST 2.0

Alcohol Smoking and Substance Involvement Screening Test - ASSIST - Teste para triagem do envolvimento com fumo, álcool e outras drogas

\begin{tabular}{|c|c|c|}
\hline I - Na sua vida, qual (is) dessas substâncias você já usou? (SOMENTE USO NÃO-MÉDICO) & NÃO & SIM \\
\hline a. Derivados do tabaco (cigarros, charuto, cachimbo, fumo de corda...) & 0 & I \\
\hline b. Bebidas alcoólicas (cerveja, vinho, destilados como pinga, uísque, vodka, vermutes...) & 0 & । \\
\hline c. Maconha (baseado, erva, haxixe...) & 0 & I \\
\hline d. Cocaína, crack (pó, pedra, branquinha, nuvem...) & 0 & I \\
\hline e. Estimulantes como anfetaminas ou ecstasy (bolinhas, rebites...) & 0 & । \\
\hline f. Inalantes (cola de sapateiro, cheirinho-da-loló, tinta, gasolina, éter, lança-perfume,benzina...) & 0 & । \\
\hline g. Hipnóticos/sedativos (remédios para dormir: diazepam, lorazepan, lorax, dienpax, rohypnol). & 0 & । \\
\hline h. Drogas alucinógenas (como LSD, ácido, chá-de-lírio, cogumelos...) & 0 & । \\
\hline i. Opióides (heroína, morfina, metadona, codeína...) & 0 & । \\
\hline j. Outras, Especificar: & 0 & I \\
\hline
\end{tabular}

\begin{tabular}{|c|c|c|c|c|c|}
\hline $\begin{array}{l}2 \text { - Durante os três últimos meses, com que freqüência você utilizou essa(s) } \\
\text { substância(s) que mencionou? (Primeira droga, depois a segunda droga, etc) }\end{array}$ & Nunca & $\begin{array}{l}\text { I ou } 2 \\
\text { vezes }\end{array}$ & Mensalmente & Semanalmente & $\begin{array}{l}\text { Diariamente ou } \\
\text { quase todo dia }\end{array}$ \\
\hline $\begin{array}{l}\text { a. Derivados do tabaco (cigarros, charuto, cachimbo, fumo de corda...) } \\
\text { b. Bebidas alcoólicas (cerveja, vinho, destilados como pinga, uísque, vodka, vermutes...) } \\
\text { c. Maconha (baseado, erva, haxixe...) } \\
\text { d. Cocaína, crack (pó, pedra, branquinha, nuvem...) } \\
\text { e. Estimulantes como anfetaminas ou ecstasy (bolinhas, rebites...) } \\
\text { f. Inalantes (cola de sapateiro, cheirinho-da-loló, tinta, gasolina, éter, lança-perfume, benzina...) } \\
\text { g. Hipnóticos/sedativos (remédios para dormir: diazepam, lorazepan, lorax, dienpax, rohypnol). } \\
\text { h. Drogas alucinógenas (como LSD, ácido, chá-de-lírio, cogumelos...) } \\
\text { i. Opióides (heroína, morfina, metadona, codeína...) } \\
\text { j. Outras, Especificar: }\end{array}$ & $\begin{array}{l}0 \\
0 \\
0 \\
0 \\
0 \\
0 \\
0 \\
0 \\
0 \\
0\end{array}$ & $\begin{array}{l}1 \\
1 \\
1 \\
1 \\
1 \\
1 \\
1 \\
1 \\
1\end{array}$ & $\begin{array}{l}2 \\
2 \\
2 \\
2 \\
2 \\
2 \\
2 \\
2 \\
2 \\
2\end{array}$ & $\begin{array}{l}3 \\
3 \\
3 \\
3 \\
3 \\
3 \\
3 \\
3 \\
3 \\
3\end{array}$ & $\begin{array}{l}4 \\
4 \\
4 \\
4 \\
4 \\
4 \\
4 \\
4 \\
4 \\
4\end{array}$ \\
\hline
\end{tabular}

\begin{tabular}{|c|c|c|c|c|c|}
\hline $\begin{array}{l}3 \text { - Durante os três últimos meses, com que freqüência você teve um forte } \\
\text { desejo ou urgência em consumir? (Primeira droga, depois a segunda droga, etc) }\end{array}$ & Nunca & $\begin{array}{l}\text { I ou } 2 \\
\text { vezes }\end{array}$ & Mensalmente & Semanalmente & $\begin{array}{l}\text { Diariamente ou } \\
\text { quase todo dia }\end{array}$ \\
\hline a. Derivados do tabaco (cigarros, charuto, cachimbo, fumo de corda...) & 0 & I & 2 & 3 & 4 \\
\hline b. Bebidas alcoólicas (cerveja, vinho, destilados como pinga, uísque, vodka, vermutes...) & 0 & I & 2 & 3 & 4 \\
\hline c. Maconha (baseado, erva, haxixe...) & 0 & l & 2 & 3 & 4 \\
\hline d. Cocaína, crack (pó, pedra, branquinha, nuvem...) & 0 & । & 2 & 3 & 4 \\
\hline e. Estimulantes como anfetaminas ou ecstasy (bolinhas, rebites...) & 0 & I & 2 & 3 & 4 \\
\hline f. Inalantes (cola de sapateiro, cheirinho-da-loló, tinta, gasolina, éter, lança-perfume,benzina...) & 0 & I & 2 & 3 & 4 \\
\hline g. Hipnóticos/sedativos (remédios para dormir: diazepam, lorazepan, lorax, dienpax, rohypnol). & 0 & । & 2 & 3 & 4 \\
\hline h. Drogas alucinógenas (como LSD, ácido, chá-de-lírio, cogumelos...) & 0 & | & 2 & 3 & 4 \\
\hline i. Opióides (heroína, morfina, metadona, codeína...) & 0 & 1 & 2 & 3 & 4 \\
\hline j. Outras, Especificar: & 0 & । & 2 & 3 & 4 \\
\hline
\end{tabular}

\begin{tabular}{|c|c|c|c|c|c|}
\hline $\begin{array}{l}4 \text { - Durante os três últimos meses, com que freqüência o seu } \\
\text { consumo de (Primeira droga, depois a segunda droga, etc) } \\
\text { resultou em problema de saúde, social, legal ou financeiro? }\end{array}$ & Nunca & $\begin{array}{l}\text { I ou } 2 \\
\text { vezes }\end{array}$ & Mensalmente & Semanalmente & $\begin{array}{l}\text { Diariamente ou } \\
\text { quase todo dia }\end{array}$ \\
\hline $\begin{array}{l}\text { a. Derivados do tabaco (cigarros, charuto, cachimbo, fumo de corda...) } \\
\text { b. Bebidas alcoólicas (cerveja, vinho, destilados como pinga, uísque, vodka, vermutes...) } \\
\text { c. Maconha (baseado, erva, haxixe...) } \\
\text { d. Cocaína, crack (pó, pedra, branquinha, nuvem...) } \\
\text { e. Estimulantes como anfetaminas ou ecstasy (bolinhas, rebites...) } \\
\text { f. Inalantes (cola de sapateiro, cheirinho-da-loló, tinta, gasolina, éter, lança-perfume,benzina...) } \\
\text { g. Hipnóticos/sedativos (remédios para dormir: diazepam, lorazepan, lorax, dienpax, rohypnol). } \\
\text { h. Drogas alucinógenas (como LSD, ácido, chá-de-lírio, cogumelos...) } \\
\text { i. Opióides (heroína, morfina, metadona, codeína...) } \\
\text { j. Outras, Especificar: }\end{array}$ & $\begin{array}{l}0 \\
0 \\
0 \\
0 \\
0 \\
0 \\
0 \\
0 \\
0 \\
0\end{array}$ & $\begin{array}{l}1 \\
1 \\
1 \\
1 \\
1 \\
1 \\
1 \\
1 \\
1 \\
1 \\
1\end{array}$ & $\begin{array}{l}2 \\
2 \\
2 \\
2 \\
2 \\
2 \\
2 \\
2 \\
2 \\
2\end{array}$ & $\begin{array}{l}3 \\
3 \\
3 \\
3 \\
3 \\
3 \\
3 \\
3 \\
3 \\
3\end{array}$ & $\begin{array}{l}4 \\
4 \\
4 \\
4 \\
4 \\
4 \\
4 \\
4 \\
4 \\
4\end{array}$ \\
\hline
\end{tabular}


Anexo I - ASSIST 2.0

Alcohol Smoking and Substance Involvement Screening Test - ASSIST - Teste para triagem do envolvimento com fumo, álcool e outras drogas

\begin{tabular}{|c|c|c|c|c|c|}
\hline $\begin{array}{l}5 \text { - Durante os três últimos meses, com que freqüência por causa do seu uso de } \\
\text { (Primeira droga, depois a segunda droga, etc) você deixou de fazer coisas que } \\
\text { eram normalmente esperadas por você? }\end{array}$ & Nunca & $\begin{array}{l}\text { I ou } 2 \\
\text { vezes }\end{array}$ & Mensalmente & Semanalmente & $\begin{array}{l}\text { Diariamente ou } \\
\text { quase todo dia }\end{array}$ \\
\hline $\begin{array}{l}\text { a. Derivados do tabaco (cigarros, charuto, cachimbo, fumo de corda...) } \\
\text { b. Bebidas alcoólicas (cerveja, vinho, destilados como pinga, uísque, vodka, vermutes...) } \\
\text { c. Maconha (baseado, erva, haxixe...) } \\
\text { d. Cocaína, crack (pó, pedra, branquinha, nuvem...) } \\
\text { e. Estimulantes como anfetaminas ou ecstasy (bolinhas, rebites...) } \\
\text { f. Inalantes (cola de sapateiro, cheirinho-da-loló, tinta, gasolina, éter, lança-perfume,benzina...) } \\
\text { g. Hipnóticos/sedativos (remédios para dormir: diazepam, lorazepan, lorax, dienpax, rohypnol). } \\
\text { h. Drogas alucinógenas (como LSD, ácido, chá-de-lírio, cogumelos...) } \\
\text { i. Opióides (heroína, morfina, metadona, codeína...) } \\
\text { j. Outras, Especificar: }\end{array}$ & $\begin{array}{l}0 \\
0 \\
0 \\
0 \\
0 \\
0 \\
0 \\
0 \\
0 \\
0\end{array}$ & $\begin{array}{l} \\
1 \\
1 \\
1 \\
1 \\
1 \\
1 \\
1 \\
1 \\
1\end{array}$ & $\begin{array}{l}2 \\
2 \\
2 \\
2 \\
2 \\
2 \\
2 \\
2 \\
2 \\
2\end{array}$ & $\begin{array}{l}3 \\
3 \\
3 \\
3 \\
3 \\
3 \\
3 \\
3 \\
3 \\
3\end{array}$ & $\begin{array}{l}4 \\
4 \\
4 \\
4 \\
4 \\
4 \\
4 \\
4 \\
4 \\
4\end{array}$ \\
\hline
\end{tabular}

\begin{tabular}{|c|c|c|c|}
\hline $\begin{array}{l}6 \text { - Há amigos, parentes ou outra pessoa que tenha demonstrado preocupação com } \\
\text { seu uso de (Primeira droga, depois a segunda droga, etc)? }\end{array}$ & NÃO, nunca & $\begin{array}{l}\text { SIM, mas não nos } \\
\text { últimos } 3 \text { meses }\end{array}$ & $\begin{array}{l}\text { SIM, nos últimos } \\
3 \text { meses }\end{array}$ \\
\hline $\begin{array}{l}\text { a. Derivados do tabaco (cigarros, charuto, cachimbo, fumo de corda...) } \\
\text { b. Bebidas alcoólicas (cerveja, vinho, destilados como pinga, uísque, vodka, vermutes...) } \\
\text { c. Maconha (baseado, erva, haxixe...) } \\
\text { d. Cocaína, crack (pó, pedra, branquinha, nuvem...) } \\
\text { e. Estimulantes como anfetaminas ou ecstasy (bolinhas, rebites...) } \\
\text { f. Inalantes (cola de sapateiro, cheirinho-da-loló, tinta, gasolina, éter, lança- perfume, benzina...) } \\
\text { g. Hipnóticos/sedativos (remédios para dormir:diazepam, lorazepan, lorax, dienpax, rohypnol...). } \\
\text { h. Drogas alucinógenas (como LSD, ácido, chá-de-lírio, cogumelos...) } \\
\text { i. Opióides (heroína, morfina, metadona, codeína...) } \\
\text { j. Outras, Especificar: }\end{array}$ & $\begin{array}{l}0 \\
0 \\
0 \\
0 \\
0 \\
0 \\
0 \\
0 \\
0 \\
0 \\
0\end{array}$ & $\begin{array}{l}1 \\
1 \\
1 \\
1 \\
1 \\
1 \\
1 \\
1 \\
1 \\
1\end{array}$ & $\begin{array}{l}2 \\
2 \\
2 \\
2 \\
2 \\
2 \\
2 \\
2 \\
2 \\
2\end{array}$ \\
\hline
\end{tabular}

\begin{tabular}{|c|c|c|c|}
\hline $\begin{array}{l}7 \text { - Alguma vez você já tentou controlar, diminuir ou parar } \\
\text { o uso de (Primeira droga, depois a segunda droga, etc)? }\end{array}$ & NÃO, nunca & $\begin{array}{l}\text { SIM, mas não nos } \\
\text { últimos } 3 \text { meses }\end{array}$ & $\begin{array}{l}\text { SIM, nos últimos } \\
3 \text { meses }\end{array}$ \\
\hline $\begin{array}{l}\text { a. Derivados do tabaco (cigarros, charuto, cachimbo, fumo de corda...) } \\
\text { b. Bebidas alcoólicas (cerveja, vinho, destilados como pinga, uísque, vodka, vermutes...) } \\
\text { c. Maconha (baseado, erva, haxixe...) } \\
\text { d. Cocaína, crack (pó, pedra, branquinha, nuvem...) } \\
\text { e. Estimulantes como anfetaminas ou ecstasy (bolinhas, rebites...) } \\
\text { f. Inalantes (cola de sapateiro, cheirinho-da-loló, tinta, gasolina, éter, lança- perfume, benzina...) } \\
\text { g. Hipnóticos/sedativos (remédios para dormir:diazepam, lorazepan, lorax, dienpax, rohypnol...). } \\
\text { h. Drogas alucinógenas (como LSD, ácido, chá-de-lírio, cogumelos...) } \\
\text { i. Opióides (heroína, morfina, metadona, codeína...) } \\
\text { j. Outras, Especificar: }\end{array}$ & $\begin{array}{l}0 \\
0 \\
0 \\
0 \\
0 \\
0 \\
0 \\
0 \\
0 \\
0 \\
0\end{array}$ & $\begin{array}{l}1 \\
1 \\
1 \\
1 \\
1 \\
1 \\
1 \\
1 \\
1 \\
1 \\
1\end{array}$ & $\begin{array}{l}2 \\
2 \\
2 \\
2 \\
2 \\
2 \\
2 \\
2 \\
2 \\
2\end{array}$ \\
\hline
\end{tabular}

\begin{tabular}{|c|c|c|c|}
\hline 8 - Alguma vez você já usou drogas por injeção? (Apenas uso não-médico)? & NÃO, nunca & $\begin{array}{l}\text { SIM, mas não nos } \\
\text { últimos } 3 \text { meses }\end{array}$ & $\begin{array}{c}\text { SIM, nos últimos } \\
3 \text { meses }\end{array}$ \\
\hline & 0 & 1 & 2 \\
\hline
\end{tabular}

Escore das questões $2.2-2.8$

\begin{tabular}{|c|c|c|c|}
\hline & Uso ocasional & Sugestivo de abuso & Sugestivo de dependência \\
\hline Tabaco & $0-3$ & $4-15$ & $16-20$ \\
\hline Álcool & $0-3$ & $4-15$ & $16-20$ \\
\hline Maconha & $0-3$ & $4-15$ & $16-20$ \\
\hline Cocaína & $0-3$ & $4-15$ & $16-20$ \\
\hline Anfetaminas & $0-3$ & $4-15$ & $16-20$ \\
\hline Inalantes & $0-3$ & $4-15$ & $16-20$ \\
\hline Sedativos & $0-3$ & $4-15$ & $16-20$ \\
\hline Alucinógenos & $0-3$ & $4-15$ & $16-20$ \\
\hline Opiáceos & $0-3$ & $4-15$ & $16-20$ \\
\hline
\end{tabular}

- Nota dos autores: após a realização deste estudo foram realizadas alterações no instrumento: a questão 7 foi reformulada, acrescentando-se " ... controlar ou parar o uso de (nome da substância) sem sucesso?

A pontuação de algumas questões foi alterada na versão 3.0 do ASSIST. Sugere-se procurar os autores da versão brasileira para uso do instrumento atualizado. 
Para análise da factibilidade do uso do ASSIST foram calculados os tempos médios de aplicação em cada grupo (atenção primária/ secundária e dependentes).

Para análise da consistência interna do instrumento foi calculado o índice de consistência interna alfa de Cronbach ${ }^{15,16}$ para álcool, tabaco, maconha e cocaína. Para análise da validade concorrente foram calculados os níveis de sensibilidade, especificidade e valores preditivos, utilizando-se como padrão-ouro o diagnóstico de abuso ou dependência determinado pelo MINI-Plus. Foram calculados os coeficientes de correlação entre os escores obtidos no ASSIST e os obtidos no AUDIT (coeficiente de Spearman) e no RTQ-S (coeficiente de Pearson).

Ética - Este projeto foi aprovado pelos comitês de ética em pesquisa da UNIFESP e da UFPR. Pessoas dos serviços de atenção primária e secundária (grupo SAPS) que receberam diagnóstico de uso abusivo ou dependência de substâncias psicoativas (escore no ASSIST > 15 para qualquer substância psicoativa, à exceção do tabaco), foram orientadas a procurar tratamento em serviços especializados, a partir de uma lista de indicações fornecida pelo entrevistador.

\section{Resultados}

A Tabela I apresenta os dados sóciodemográficos da amostra estudada, dividida de acordo com os grupos de atenção primária/ secundária (SAPS) e de dependentes de drogas (DEP). Os grupos foram semelhantes em relação a todas as características sociodemográficas ( $p>0,05)$.

Considerando-se a amostra total, o tempo mínimo de aplicação do ASSIST foi de I minuto e o máximo de 30, sendo a mediana do tempo gasto de 6 minutos (Intervalo interquartil de 2 minutos). O tempo de aplicação nos grupos SAPS e DEP foi semelhante (respectivamente $7 \pm 5$ minutos, $p>0,05)$.

As médias de escore máximo do ASSIST, incluindo álcool e tabaco, foram $11 \pm 6$ na amostra total, sendo $8 \pm 5$ no grupo SAPS e 16 \pm 3 no grupo DEP $(p<0,05)$. A Tabela 2 apresenta as médias dos escores do ASSIST para cada droga nos dois grupos e na amostra total. Apenas 5, I\% dos pacientes da amostra total não atingiram pontuação (escore do ASSIST=0), sendo todos do grupo SAPS.

\begin{tabular}{|c|c|c|c|c|}
\hline Características & & $\begin{array}{l}\text { SAPS }^{\#} \\
(n=99)\end{array}$ & $\begin{array}{l}\text { Dependentes } \\
\quad(n=48)\end{array}$ & $\begin{array}{l}\text { TOTAL } \\
(\mathrm{n}=147)\end{array}$ \\
\hline & Ambulatório de álcool e drogas & 0 & 16,7 & 5,5 \\
\hline \multirow{6}{*}{ Local de recrutamento } & Internação de álcool e drogas & 0 & 83,3 & 27 \\
\hline & Ambulatório clínico ou consultório médico & 67,7 & 0 & 45,5 \\
\hline & Internação clínica geral & 18,2 & 0 & 12,5 \\
\hline & Pronto-Socorro & | & 0 & I \\
\hline & $\begin{array}{l}\text { Ambulatório ou internação } \\
\text { psiquáátricos }\end{array}$ & 2 & 0 & 7 \\
\hline & Outros & 8,1 & 0 & । \\
\hline \multirow[t]{5}{*}{ Idade } & Faixas etárias (\%) & & & \\
\hline & I8-25anos & 42 & 40 & 41.5 \\
\hline & 26-35anos & 30 & 31 & 30,5 \\
\hline & $36-45$ anos & 27 & 29 & 28 \\
\hline & idade média ( \pm desvio padrão) & $29 \pm 8$ & $30 \pm 8$ & $29 \pm 8$ \\
\hline Sexo & $\begin{array}{l}\text { Masculino } \\
\text { Feminino }\end{array}$ & $\begin{array}{l}62,6 \\
37,4\end{array}$ & $\begin{array}{l}60,4 \\
39,6\end{array}$ & $\begin{array}{l}62 \\
38\end{array}$ \\
\hline \multirow[t]{6}{*}{ Estado civil } & Casado & 26,3 & 20,8 & 24,5 \\
\hline & Amasiado & 20,2 & 14,6 & 18 \\
\hline & Separado & 5,1 & 4,2 & 5 \\
\hline & Viúvo & 0 & 2,1 & 0,5 \\
\hline & Divorciado & 6,1 & 4,2 & 5,5 \\
\hline & Nunca se casou & 42,4 & 54,2 & 46,5 \\
\hline \multirow[t]{5}{*}{ Moradia } & Casa própria ou da familila & 80,8 & 72,9 & 78 \\
\hline & Residência alugada & 17,2 & 22,9 & 19.5 \\
\hline & Abrigo & 0 & 2,1 & 0.5 \\
\hline & Sem endereço fixo & । & 0 & 0.5 \\
\hline & Outros & | & 2,1 & 1.5 \\
\hline \multirow[t]{5}{*}{ Raça } & Branca & 66,7 & 79,2 & 71 \\
\hline & Amarela & 0 & 4,2 & 1,5 \\
\hline & Outras & 2,0 & 0 & 1,5 \\
\hline & Negro & 1,0 & 4,2 & 2 \\
\hline & Mulato & 30,3 & 12,5 & 24 \\
\hline
\end{tabular}

\# SAPS (serviços de atenção primária e secundária à saúde) \& DEPS (Dependentes); Não há diferença siggificativa entre os dois grupos

Nenhum paciente do grupo de dependentes apresentou escore zero no ASSIST. Como pode ser observado na Tabela 2, no grupo SAPS os maiores escores no ASSIST (droga principal) foram os relativos a álcool (33,3\%), tabaco (50,9\%) e maconha (5,5\%). No grupo de dependentes, os maiores escores foram os relativos a álcool (53, l\%), cocaína (24,5\%) e tabaco (12,2\%). Nenhum dos entrevistados pontuou no ASSIST em relação a alucinógenos ou a opiáceos. Se fossem excluídos os escores de álcool e tabaco, as médias dos escores do ASSIST passariam a ser $4 \pm 6$ na amostra total, $2 \pm 4$ no grupo SAPS e $9 \pm 8$ no grupo de dependentes, sendo que $62,6 \%$ dos pacientes do grupo SAPS e $29,1 \%$ do grupo de dependentes pontuariam zero.

A distribuição dos escores no ASSIST, por faixas de risco, está apresentada na Tabela 3. No grupo SAPS, 28\% dos pacientes se encontravam abaixo da faixa de risco ( 0 a 3), $66 \%$ na faixa de risco (4 a I 5 pontos) e $6 \%$ com escore sugestivo de dependência $(>\mid 5)$, tendo sido encaminhados para serviços especializados. No grupo DEP nenhum paciente pontuou abaixo da faixa de risco, 27\% pontuaram na faixa de risco (4 a 15 pontos) e $63 \%$ na faixa sugestiva de dependência ( $>15)$.

A Tabela 4 apresenta a porcentagem de respostas positivas para cada questão. As duas primeiras questões, que abordam o uso na vida, e a freqüência de uso atual (últimos três meses), foram as que apresentaram maior proporção de respostas positivas. Seguem-se a ela, as questões 7 e 8 , que abordam, respectivamente, a preocupação de pessoas próximas 
Tabela 2 - Escores médios do ASSIST para cada droga (média \pm desvio padrão), incluindo e excluindo álcool e tabaco.

Porcentagem de pacientes da amostra total para os quais o escore máximo atingido foi naquela classe de drogas

\begin{tabular}{|c|c|c|c|c|c|c|c|c|c|}
\hline \multirow[t]{2}{*}{ Tipo de droga } & \multicolumn{3}{|c|}{ SAPS } & \multicolumn{3}{|c|}{$\begin{array}{c}\text { Dependentes de substâncias } \\
\text { psicoativas }\end{array}$} & \multicolumn{3}{|c|}{ Total } \\
\hline & Média $\pm d p$ & $\begin{array}{c}\% \\
\text { incluindo } \\
\text { álcool e } \\
\text { tabaco }\end{array}$ & $\begin{array}{c}\% \\
\text { excluindo } \\
\text { álcool e } \\
\text { tabaco }\end{array}$ & Média $\pm d p$ & $\begin{array}{c}\% \\
\text { incluindo } \\
\text { álcool e } \\
\text { tabaco }\end{array}$ & $\begin{array}{c}\% \\
\text { excluindo } \\
\text { álcool e } \\
\text { tabaco }\end{array}$ & Média $\pm d p$ & $\begin{array}{c}\% \\
\text { incluindo } \\
\text { álcool e } \\
\text { tabaco }\end{array}$ & $\begin{array}{c}\% \\
\text { excluindo } \\
\text { álcool e } \\
\text { tabaco }\end{array}$ \\
\hline $\begin{array}{l}\text { Tabaco } \\
\text { Álcool } \\
\text { Maconha } \\
\text { Cocaína } \\
\text { Anfetaminas } \\
\text { Inalantes } \\
\text { Sedativos } \\
\text { Não consome } \\
\text { nenhumadroga }\end{array}$ & $\begin{array}{c}6,5 \pm 5,7 \\
4,1 \pm 4,7 \\
1,8 \pm 3,7 \\
0,5 \pm 1,8 \\
0,3 \pm 0,2 \\
0,1 \pm 0,6 \\
0 \pm 0,2\end{array}$ & $\begin{array}{c}50,9 \\
33,3 \\
5,5 \\
0,9 \\
0,9 \\
- \\
0,9 \\
7,4\end{array}$ & $\begin{array}{c}- \\
- \\
28,3 \\
6,1 \\
1,0 \\
- \\
1,0 \\
67,7\end{array}$ & $\begin{array}{c}8,3 \pm 5,4 \\
\mid 1,9 \pm 6,6^{*} \\
5,1 \pm 6,1^{*} \\
6,9 \pm 7,7^{*} \\
0,8 \pm 2,6 * \\
1,2 \pm 4,1^{*} \\
1,4 \pm 4,2^{*}\end{array}$ & $\begin{array}{c}12,2 \\
53,1 \\
4,1 \\
24,5 \\
- \\
4 \\
4,1 \\
0\end{array}$ & $\begin{array}{c}- \\
- \\
22,9 \\
39,6 \\
2,08 \\
6,25 \\
4,17 \\
31,3\end{array}$ & $\begin{array}{l}7,1 \pm 5,7 \\
6,7 \pm 6,5 \\
2,9 \pm 4,8 \\
2,6 \pm 5,5 \\
0,3 \pm 1,5 \\
0,5 \pm 2,4 \\
0,5 \pm 2,5\end{array}$ & $\begin{array}{c}38,8 \\
39,5 \\
5,1 \\
8,3 \\
0,6 \\
0,6 \\
1,9 \\
5,1\end{array}$ & $\begin{array}{c}24,5 \\
\mid 4,9 \\
1,4 \\
6,1 \\
3,4 \\
49,7\end{array}$ \\
\hline
\end{tabular}

* difere do grupo SAPS ( $p<0,05)$; Obs: Alguns participantes atingiram os mesmos escores máximos para mais de uma droga.

Tabela 3 - Distribuição dos escores do ASSIST, segundo as faixas de risco para desenvolvimento de abuso ou dependência. Porcentagem de pessoas da amostra total $(\mathrm{N}=147)$

\begin{tabular}{|c|c|c|c|c|c|c|}
\hline Tipo de droga & $\begin{array}{l}\text { Escore=0 } \\
\text { abstêmios }\end{array}$ & $\begin{array}{l}\text { Escore de } \\
\text { I a } 3 \\
\text { abaixo da } \\
\text { faixa de }\end{array}$ & $\begin{array}{l}\text { Escore } \\
4-15 \\
\text { faixa de } \\
\text { risco }\end{array}$ & $\begin{array}{c}\text { Escore de } \\
16-20 \\
\text { sugestivo de } \\
\text { dependência }\end{array}$ & $\begin{array}{c}\text { Escore } \\
\text { média } \pm \text { SD } \\
\text { (incluindo } \\
\text { escores }=0 \text { ) }\end{array}$ & $\begin{array}{c}\text { Escore } \\
\text { média } \pm \text { SD } \\
\text { (excluindo } \\
\text { escores }=0 \text { ) }\end{array}$ \\
\hline $\begin{array}{l}\text { Tabaco } \\
\text { Álcool } \\
\text { Maconha } \\
\text { Cocáina } \\
\text { Anfetaminas } \\
\text { Inalantes } \\
\text { Sedativos } \\
\text { Alucinógenos } \\
\text { Opiáceos }\end{array}$ & $\begin{array}{c}30 \\
18 \\
61,5 \\
71 \\
93 \\
90,5 \\
92 \\
94 \\
98\end{array}$ & $\begin{array}{c}7 \\
27,5 \\
12,5 \\
9 \\
5.5 \\
6 \\
5 \\
3 \\
2\end{array}$ & $\begin{array}{c}61 \\
39,5 \\
23,5 \\
12 \\
0,7 \\
2 \\
2 \\
3 \\
0\end{array}$ & $\begin{array}{c}2 \\
15 \\
3 \\
8,5 \\
0,7 \\
1,5 \\
1,5 \\
1,5 \\
0\end{array}$ & $\begin{array}{l}7,0 \pm 5,3 \\
6,8 \pm 6,5 \\
2,8 \pm 4,8 \\
2,6 \pm 5,5 \\
0,2 \pm 1,5 \\
0,7 \pm 2,3 \\
0,6 \pm 2,4 \\
0,4 \pm 1,6 \\
0,03 \pm 0,2\end{array}$ & $\begin{array}{l}8,3 \pm 4,7 \\
8,2 \pm 6,3 \\
7,4 \pm 5,1 \\
9,1 \pm 6,9 \\
3,9 \pm 4,7 \\
2,3 \pm 4,0 \\
4,0 \pm 5,3 \\
2,6 \pm 3,5 \\
1,3 \pm 0,6\end{array}$ \\
\hline
\end{tabular}

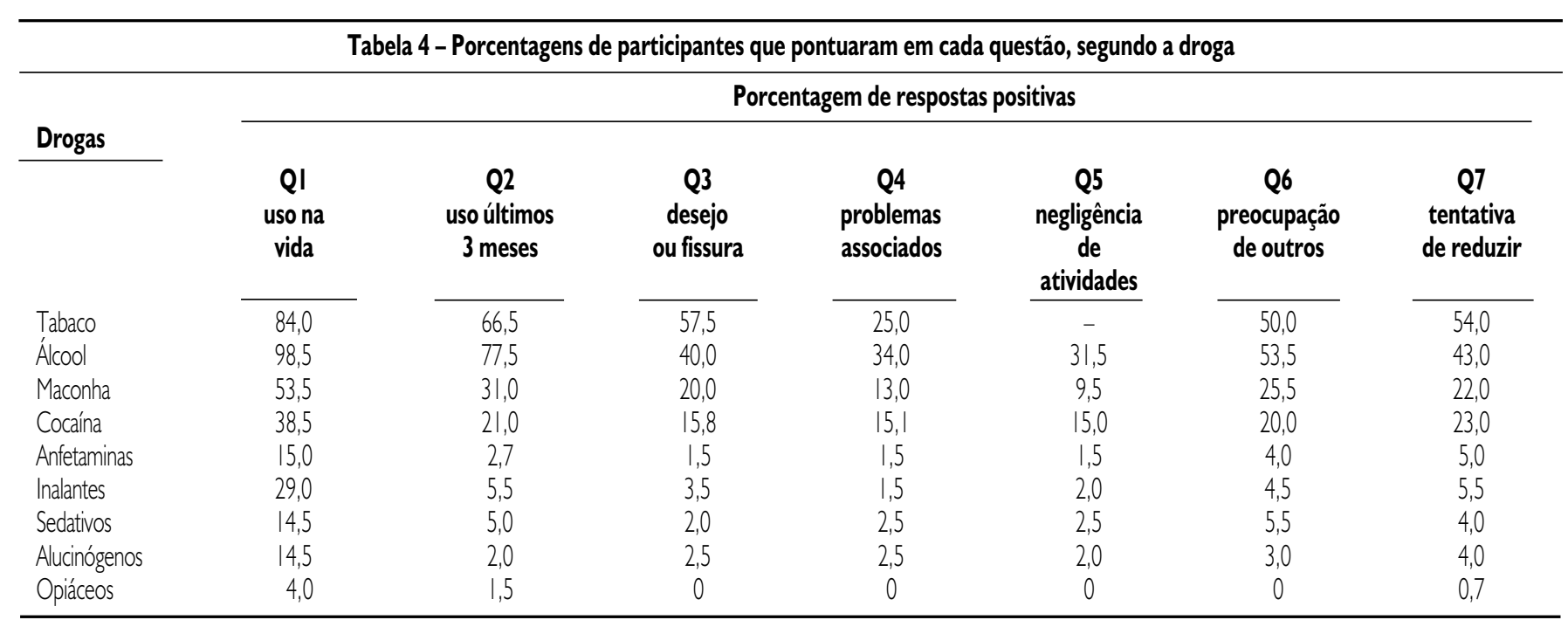




\section{Tabela 5 - Índices de validade concorrente do ASSIST, considerando-se} como padrão-ouro o diagnóstico pelo MINI-Plus

\begin{tabular}{|c|c|c|c|c|}
\hline & Sensibilidade & Especificidade & VPPI & $\mathrm{VPN}^{2}$ \\
\hline $\begin{array}{l}\text { Álcool } \\
\text { Cocaína } \\
\text { Maconha }\end{array}$ & $\begin{array}{l}91 \\
84 \\
87\end{array}$ & $\begin{array}{l}79 \\
98 \\
95\end{array}$ & $\begin{array}{l}80 \\
93 \\
87\end{array}$ & $\begin{array}{l}91 \\
96 \\
85\end{array}$ \\
\hline
\end{tabular}

$\mathrm{VPP}=$ Valor Preditivo Positivo; ${ }^{2} \mathrm{VPN}=$ Valor Preditivo Negativo

com o uso e as tentativas de redução do uso. O uso de drogas por via endovenosa (questão 8) foi relatado por 10,5\% da amostra total, sendo $23 \%$ dos pacientes do grupo DEP e $4 \%$ dos pacientes do grupo SAPS.

O pequeno número de usuários de alguns tipos de drogas não permitiu o cálculo de confiabilidade para todas as drogas. A consistência interna do instrumento, avaliada pelo coeficiente alfa de Cronbach, foi considerada boa, sendo os coeficientes: 0,80 para álcool, 0,80 para tabaco, 0,79 para maconha e 0,81 para cocaína.

A Tabela 5 apresenta os valores de sensibilidade, especificidade e valores preditivos positivo e negativo do ASSIST, considerandose como padrão-ouro o diagnóstico de abuso ou dependência atual pelo MINI-Plus. Foram realizados cálculos para as três drogas mais prevalentes: álcool, maconha e cocaína. O instrumento mostrou bons índices de sensibilidade (variando de $84 \%$ a $91 \%$ ), especificidade (de $79 \%$ a $98 \%$ ), valores preditivos positivos (80\% a $93 \%$ ) e negativos (85\% a $96 \%)$. É importante salientar que estes valores preditivos se aplicam aos índices de prevalência da amostra estudada, podendo sofrer alterações em populações com diferentes índices de prevalência.

Em relação aos usuários de álcool, foi encontrada correlação significativa entre os escores do ASSIST e os escores do AUDIT $(r=0,73 p<0,00 I)$. Quanto ao uso do tabaco, foi observada uma correlação significativa entre os escores obtidos no ASSIST e os do RTQ-S (Fargerstron), sendo o coeficiente de correlação de 0,9l $(p<0,001)$.

\section{Discussão}

Os dados deste estudo indicam que 0 ASSIST pode ser um instrumento útil na triagem do uso abusivo de álcool e outras drogas em serviços de atenção primária à saúde. $\mathrm{A}$ versão brasileira do instrumento apresentou boa sensibilidade e especificidade para álcool, maconha e cocaína. O tempo necessário para sua aplicação não foi excessivo, permitindo sua incorporação à anamnese de rotina.

Na população estudada, o álcool e tabaco se confirmaram como as principais substâncias legalizadas causadoras de abuso, com prevalências expressivas, ressaltando a importância da abordagem do tema junto à população. Maconha e cocaína foram as principais substâncias ilícitas causadoras de problemas na amostra estudada. O fato de 34\% da amostra total reconhecer ter problemas de saúde e sociais associados ao uso de álcool enfatiza a influência do abuso de álcool na saúde da população, considerada pela OMS a principal causa evitável de morbidade e mortalidade ${ }^{6}$.

O ASSIST é proposto como um instrumento a ser usado em ações de prevenção primária e, principalmente, secundária do abuso de substâncias psicoativas. Programas de prevenção secundária se propõem a detectar precocemente pessoas que não tenham atingido estágios avançados dos transtornos de uso de substâncias psicoativas, mas que apresentam uso potencialmente de risco. Podem visar também o aumento da consciência dos pacientes a respeito do problema e incentivar mudanças de comportamento.

Os clínicos gerais e outros profissionais de saúde têm, potencialmente, um importante papel na detecção precoce do abuso de substâncias psicoativas ${ }^{17}$. Embora, na teoria, seja reconhecida a necessidade de equilíbrio entre as atuações assistenciais e preventivas, em geral a formação dos profissionais é muito mais voltada para a assistência do que para a prevenção. É importante fornecer aos profissionais de saúde treinamento e instrumentos adequados para que eles possam, de fato, atuar na prevenção primária e secundária do uso abusivo de álcool e outras drogas. A modificação de comportamentos alcançada com medidas de prevenção pode trazer benefícios não só para a saúde das pessoas, mas também economizar gastos do setor saúde ${ }^{18}$.
O uso de instrumentos padronizados auxilia o profissional na determinação de critérios diagnósticos e nos procedimentos a serem utilizados. Uma das maiores dificuldades dos profissionais de saúde, em relação ao uso de álcool de seus pacientes, consiste na determinação dos limites, a partir dos quais, o uso pode ser considerado abusivo ${ }^{19}$. Neste aspecto, é clara a utilidade do ASSIST, pois seu resultado permite classificar o padrão de uso, facilitando os procedimentos de intervenção ou encaminhamento.

O uso de instrumentos de triagem na rotina de unidades de atenção primária e secundária pode também ser útil na identificação dos principais problemas de determinada comunidade, auxiliando o planejamento de políticas de saúde. Basséres \& Lins (199| $)^{20}$ ressaltaram a importância de as ações nos serviços de atenção primária serem adequadas às características e cultura locais para aumentar a eficácia do trabalho. Além disso, o uso de instrumentos padronizados pode facilitar a avaliação das intervenções e tratamentos oferecidos para a população estudada ${ }^{21}$.

De modo geral, o uso do ASSIST se mostrou bastante promissor, mas é preciso considerar que ele foi utilizado em situação "ideal" de uso, tendo sido aplicado por pesquisadores com alto nível de motivação. Além disso, a resposta obtida pelo uso de um instrumento em situações de pesquisa formal, como ocorre em estudos de validação, pode levar o paciente a respostas mais sinceras. Tal franqueza pode não ser encontrada em outras circunstâncias ${ }^{22}$. A aplicação do instrumento em um protocolo de pesquisa pode também ter aumentado seu tempo de administração, uma vez que os pesquisadores não estavam, no momento da entrevista, sob as pressões de horário a que o clínico é usualmente submetido em sua rotina diária. Portanto, é possível que seu tempo de aplicação possa ser reduzido quando incorporado a uma consulta clínica.

Percebe-se, então, a importância de avaliar o desempenho do instrumento em situações naturais. Encontra-se em andamento um estudo no qual o ASSIST será utilizado por profissionais de saúde na rede pública. Se comprovado seu bom desempenho e confiabilidade, ele poderá se tornar muito útil na detecção e intervenção precoce junto a usuários de substâncias psicoativas. 


\section{Conclusões}

A versão brasileira ASSIST apresentou boa sensibilidade, especificidade, consistência interna e validade, sugerindo sua utilidade na detecção do uso abusivo de álcool e outras substâncias psicoativas. Entretanto, faz-se necessária a sua testagem quando utilizado por profissionais de saúde em situações cotidianas de assistência primária e secundária à saúde.

\section{Agradecimentos}

Este trabalho contou com o suporte financeiro da Organização Mundial de Saúde (OMS) e da Associação Fundo de Incentivo à Psicofarmacologia (AFIP).

\section{Conflito de interesse: não há}

\section{SUMMARY}

Validation of the Brazilian version of Alcohol, Smoking and Substance InVOlVement Screening TeSt (ASSIST)

BACKGROUND. Considering the increasing levels of psychoactive substance use in Braziland around the world, it is necessary the development of an instrument to early detection, that could be considered valid, reliable and useful at primary health care settings. In order to do that, an international team of researchers, supported by the World Health Organization (WHO) developed the Alcohol Smoking and Substance Screening Test (ASSIST). The objective of the present study was to evaluate the psychometric properties of the Brazilian version of ASSIST, as well as its reliability and concurrent validity in the detection of psychoactive substance use and related problems.

METHODS. The ASSIST and three validated diagnostic instruments (Mini-Plus, AUDIT, RTQ) were applied to 99 patients of primary/ secondary health care services and to 48 patients in treatment for alcohol and drug dependence in specialized services, at São Paulo and Curitiba cities.

RESULTS. The ASSIST'scorestoalcoholshowed goodcorrelation with AUDIT'scores. The ASSIST presented high sensitivity and specificity in the detection of alcohol, cannabis and cocaine abuse/dependence, considering the MINI-Plus diagnostic the "gold-standard". The reliability of the instrument was good (Cronbach's alpha of 0.80 to alcohol, 0.79 to cannabis and 0.81 to cocaine).

COnclusions. The psychometricproperties of the Brazilian version of the ASSIST seem to be satisfactory, recommending its use in patients of primary/secondary health care services. [Rev Assoc Med Bras 2004; 50(2): 199-206]

KEY WORDS: ASSIST-OMS. Validation. Drug dependence. Screening test. Alcohol.

\section{REFERÊNCIAS}

I. Galduróz JCF, Carlini EA, Noto AR, Napo SA. I Levantamento domiciliar nacional sobre o uso de drogas psicotrópicas. Parte A: estudo envolvendo as 24 maiores cidades do estado de São Paulo - São Paulo: CEBRID-Centro Brasileiro de Informações sobre drogas Psicotrópicas: UNIFESP- Universidade Federal de São Paulo; 2000.

2. Carlini E A, Galduróz JCF, Noto AR, Nappo SA. Levantamento domiciliar sobre o uso de drogas psicotrópicas no Brasil. São Paulo: CEBRID/SENAD; 2001.

3. Galduróz JCF, Noto AR, Napo SA. IV Levantamento de uso de drogas psicotrópicas em estudantes Brasileiros- 1997.São Paulo: CEBRID-Centro Brasileiro de Informações sobre drogas Psicotrópicas: UNIFESP-Universidade Federal de São Paulo; 1997.

4. Galduróz JCF, Noto AR, Nappo SA, Carlini ELA. First household survey on drug abuse in São Paulo, Brazil, 1999: principal findings. São Paulo Med J 2003; I2I(6):23 I-7.

5. United Nations International Drug Control Program - 1997. World Drug Report. New York: Oxford University Press; 1997.

6. WHO ASSIST Working Group. The alcohol, Smoking and substance Involvement Screening Test (ASSIST): development, reliability and feasibility. Addiction 2002; 97: I 183-94.

7. Formigoni MLOS, organizador. A intervenção breve na dependência de drogas: a experiência brasileira. São Paulo: Contexto; 1992.

8. Masur J, Monteiro MG. Validation of the CAGE, alcoholism screening test in brazilian psychiatric inpatient setting. Braz J Med Biol 1983; 16:215-8

9. Méndez EB. Uma Versão Brasileira do AUDIT (Alcohol Use Disorders Identification Test) [dissertação]. Pelotas: Faculdade de Medicina, Universidade Federal de Pelotas; 1999.

10. Mendoza-Sassi RA, Béria JU Prevalence of alcohol use disorders and associated factors: a population-based study using AUDIT in southern Braz Addiction 2003; 98:799-804.

II. Jorge MR, Masur J. Questionários padronizados para avaliação do grau de severidade da síndrome de dependência do álcool. J Bras Psiquiatria 1986; 35:287-92.

12. Sheehan DV, Lecrubier Y, Sheehan KH, Amorim $P$, Janavs J, Weiller E, Hergueta $T$, Baker R, Dunbar GC. The Mini-International Neuropsychiatric Interview (M.I.N.I.): the development and validation of a structured diagnostic psychiatric interview for DSM-IV and ICD-10. J Clin Psychiatr 1998; 59(20):22-33.

13. Amorim P. Mini International Neuropsychiatric Interview (MINI): validação de entrevista breve para diagnóstico de transtornos mentais. Rev Bras Psiquiatria 2000; 22:106-15.

14. Tate JC, Schmitz JM. A proposed revision of the Fagerstrom Tolerance Questionnaire. Addict Behav 1993; | 8: 135-43.

I5. Cronbach LJ. Coefficient alpha and the internal structure of tests. Psychometrika, 1951; 1 6:297-335.

16. Portney LG, Watkins MP. Foundations of Clinical Research. Applications to practice. Norwlalk: Applenton and Lange; 1993. p. 69-85.

17. Kappa-Larson P, Lathrop L. Early detection and intervention for hazardous ethanol use. Nurse Practitioner 1993; | 8: 50-5.

18. Berlinguer G. Ética da prevenção. In: Ética da saúde. São Paulo: Editora Hucitec; 1996.

19. Sanchez-Craig M. How much is too much? Estimates of hazardous drinking based on client's choice self-report. Br J Addiction 1986; $81: 251-6$

20. Basséres MF, Lins PH. Atenção primária: uma experiência acumulada. In: Baptista M, Inem C, organizadores. Toxicomanias: abordagem multidisciplinar. NEPAD/UERJ, Rio de Janeiro: Editora Sette Letras; 1997. P 75-6.

21. Duarte CS, Bodrim IAS. Instrumentos de avaliação. Rev Bras Psiquiatria 2000; 22:55-8.

22. Hesselbrock M, Babor TF, Hesselbock V, Meyer RE, Workman K. Never Believe an alcoholic? On the validity of self-report measures of alcohol dependence and related constructs. Int J Addictions 1 983; । 8:593-609.

Artigo recebido: 17/06/03

Aceito para publicação: 09/12/03 